\title{
Mitochondrial transcription factor $A$ is the major protein in rodent hepatocytes that recognizes DNA lesions induced by $\mathrm{N}$-acetoxy-acetylaminofluorene
}

\author{
Monika Pietrowska $^{1 \bowtie}$, Izabela Kołodziejczyk ${ }^{1}$ and Piotr Widłak ${ }^{1,2}$ \\ ${ }^{1}$ Department of Experimental and Clinical Radiobiology, Maria Skłodowska-Curie Memorial Cancer Center and \\ Institute of Oncology, Gliwice, Poland, and ${ }^{2}$ Faculty of Biotechnology, University of Rzeszow, Werynia, Poland; \\ $\square_{e-m a i l:}$ __pietrowska@io.gliwice.pl
}

Received: 21 September, 2006; revised: 06 November, 2006; accepted: 23 November, 2006

available on-line: 04 December, 2006

\begin{abstract}
Extracts from rodent liver cells contain an abundant protein that recognizes DNA adducts induced by the chemical carcinogen $\mathrm{N}$-acetoxy-acetylaminofluorene (AAAF). This protein also has a strong affinity for DNA damaged by cisplatin (DDP), but not by benzo(a)pyrene diolepoxide or UV-radiation, and has been termed AAAF/DDP-DDB. Here we purified this protein from rat tissue and analyzed it by mass spectrometry and identified it as mitochondrial transcription factor A (TFAM). Experiments with bacterially expressed recombinant TFAM confirmed its high affinity for DNA damaged by AAAF. Assuming its abundance and specificity for AAAF induced lesions, TFAM may significantly impede recognition and repair of DNA adducts induced by AAAF and other derivatives of 2-aminofluorene.
\end{abstract}

Keywords: AAAF, cisplatin, TFAM, UV-radiation

The first step of each DNA repair mechanism is damage recognition carried out by different protein detectors, which depend on the type of damage and repair pathway. Among detector complexes involved in NER, an important and universal pathway which removes a broad spectrum of DNA damage, are XPC/HR23B, UV-DDB (XPE) and XPA/ RPA (reviewed in Wood, 1999; Sancar et al., 2004). Many other proteins that bind DNA lesions repaired by NER have been detected in different organisms, but their relevance to DNA repair remains elusive. Among these damaged DNA-binding (DDB) proteins is chromatin protein HMGB1 (formerly termed HMG-1) and other HMG-box-containing proteins, which were found to have a high affinity for 1,2intrastrand crosslinks induced in DNA by cis-diaminedichloroplatinum (cis-DDP, cisplatin) (Pil \& Lippard, 1992) and other bulky DNA damage (reviewed in Widłak et al., 2006). It has been proposed that HMG-box-containing proteins may compete with DNA repair proteins, making repair less efficient and facilitating apoptosis induced by cis-DDP and other DNA-damaging factors; this was called the damage-shielding model (reviewed in Widłak et al., 2006; Reeves \& Adair, 2006).

Derivatives of the aromatic amine 2-aminofluorene (2-AF) are known hepatocarcinogens and the formation of covalent DNA adducts seems to be an essential step in carcinogenesis induced by these compounds (Kroese et al., 1988; Poirier et al., 1991). $\mathrm{N}$-acetoxy-acetylaminofluorene (AAAF), which induces mostly the dG-C8-AAF form of adduct, is among the best studied of such compounds (reviewed in Heflich \& Neft, 1994). Although DNA adducts induced by derivatives of 2-AF are removed by NER, some aspects of their repair differ from that of other bulky DNA adducts. Adducts induced by AAAF were not preferentially removed from transcriptionally active genes (Tang et al., 1989), and their removal was not biased toward the tran-

Abbreviations: AAAF, N-acetoxy-acetylaminofluorene; BPDE, benzo(a)pyrene diolepoxide; cis-DDP, cis-diamine-dichloroplatinum, cisplatin; DDB, damaged-DNA binding (protein); EMSA, electrophoretic mobility shift assay; HMG, high mobility group (protein); MNase, micrococcal nuclease; mtDNA, mitochondrial DNA; mtTFA, mitochondrial transcription factor A; NER, nucleotide excision repair; PAGE, polyacrylamide gel electrophoresis; TFAM, mitochondrial transcription factor A; 2-AF, 2-aminofluorene. 
scribed strand (McGregor et al., 1997). In contrast to DNA lesions induced by either UV-radiation or benzo(a)pyrene, adducts induced by 2-AF derivatives were not preferentially removed from matrix attached DNA (Widłak \& Rzeszowska-Wolny, 1999). It seems probable that structural properties of AAAF-induced damage and/or specific mechanisms of such damage recognition contribute to low efficiency of repair of DNA adducts induced by AAAF.

We have previously shown that HMGB1 protein has elevated affinity to DNA damaged by AAAF (Łanuszewska \& Widłak, 2000). Two additional proteins that specifically bind DNA damaged by AAAF have also been detected in nuclear extracts from rat liver cells (Rzeszowska-Wolny \& Widłak, 1999; Pietrowska et al., 2000). Major protein detected in extracts from rat and mouse liver cells that preferentially binds to DNA damaged by AAAF has an apparent molecular mass of about $25 \mathrm{kDa}$. This abundant protein (at least $10^{5}$ molecules per cell) also has a strong affinity for DNA damaged by cisplatin, but not by BPDE or UV-radiation, and was termed AAAF/DDP-DDB (Pietrowska \& Widłak, 2005). Here we aimed to isolate and identify this putatively novel DDB protein. The AAAF/DDP-DDB protein has been chromatographically purified from rat liver cells and identified by mass spectrometry as mitochondrial transcription factor A (TFAM, mtTFA), which was verified using bacterially expressed recombinant protein for in vitro DNA-binding assays.

\section{MATERIALS AND METHODS}

Preparation of fractions from rat liver cells. Liver tissue of adult male Wistar rats was mechanically homogenized on ice in buffer consisting of 10 $\mathrm{mM}$ Hepes/NaOH (pH 7.9), $10 \mathrm{mM} \mathrm{KCl}, 1.5 \mathrm{mM}$ $\mathrm{MgCl}_{2}, 0.1 \mathrm{mM}$ EGTA, $0.5 \mathrm{mM}$ DTT, $2.2 \mathrm{M}$ sucrose, $1 \%$ Nonidet P40 and a protease inhibitor mixture (Complete $^{\mathrm{TM}}$, Boehringer). The homogenate was centrifuged for $10 \mathrm{~min}$ at $400 \times \mathrm{g}$ and washed in the same buffer without detergent to obtain the fraction of cell nuclei. To obtain nuclear extracts nuclei were incubated for $30 \mathrm{~min}$ on ice with a buffer consisting of $10 \mathrm{mM}$ Hepes/ $\mathrm{NaOH}(\mathrm{pH} 7.9), 1.5 \mathrm{mM} \mathrm{MgCl}{ }_{2}$, $0.1 \mathrm{mM}$ EGTA, $0.5 \mathrm{mM}$ DTT, 5\% glycerol, $10 \mathrm{mM}$ $\mathrm{KCl}$ and $0.4 \mathrm{M} \mathrm{NaCl}$, and then insoluble remnants were pelleted by centrifugation for $30 \mathrm{~min}$ at 16000 $\times g$ at $4^{\circ} \mathrm{C}$. The chromatin fraction was purified from rat liver nuclei treated with micrococcal nuclease (MNase). Briefly, nuclei suspended in $80 \mathrm{mM}$ phosphate buffer ( $\mathrm{pH}$ 6.8) containing $5 \mathrm{mM} \mathrm{CaCl}_{2}, 1 \mathrm{mM}$ DTT and $350 \mathrm{mM} \mathrm{NaCl}$ were incubated with MNase at $37^{\circ} \mathrm{C}$ until about $50 \%$ of chromatin was cleaved into mononucleosomal fragments. After the nuclease digestion, the reaction mixture was diluted twofold with $350 \mathrm{mM} \mathrm{NaCl}$ in $80 \mathrm{mM}$ phosphate buffer $(\mathrm{pH}$ 6.8) and EDTA (final conc. $2 \mathrm{mM}$ ), and incubated on ice for $10 \mathrm{~min}$, then insoluble remnants were pelleted by centrifugation for $10 \mathrm{~min}$ at $16000 \times \mathrm{g}$ at $4^{\circ} \mathrm{C}$. Protein concentration was assayed with the Protein Assay Kit (BioRad). Proteins were resolved on 13\% polyacrylamide/SDS gels and stained with silver or Coomassie Brilliant Blue.

Purification of AAAF/DDP-DDB protein. A chromatin fraction isolated from liver cells was subjected to chromatography on hydroxyapatite (type I, BioRad). Chromatin was applied onto the column equilibrated with buffer consisting of $160 \mathrm{mM}$ phosphate buffer (pH 6.8), $1 \mathrm{mM}$ DTT and $350 \mathrm{mM}$ $\mathrm{NaCl}$, and then the column was washed with the same buffer. Protein fractions were eluted with a linear $0.4-2 \mathrm{M} \mathrm{NaCl}$ gradient in $160 \mathrm{mM}$ phosphate buffer ( $\mathrm{pH}$ 6.8), $1 \mathrm{mM}$ DTT. Selected fractions were diluted with $160 \mathrm{mM}$ phosphate buffer and $1 \mathrm{mM}$ DTT until $150 \mathrm{mM} \mathrm{NaCl}$ was reached, and then applied onto a CM-Sephadex column pre-equilibrated with the same buffer. Column-bound proteins were eluted with $0.2,0.6$ and $1.0 \mathrm{M} \mathrm{NaCl}$ in phosphate buffer. The activity of AAAF/DDP-DDB protein was analyzed in chromatographic fractions using EMSA and Southwestern-blot assays (see below).

Purification of recombinant TFAM protein. Six-His-tagged rat recombinant TFAM was expressed in an Escherichia coli bacterial expression system and purified using chromatography on Ni-NTA agarose. cDNA for the mature form of rat hepatoma TFAM (residues 43-246; without MLR domain) was cloned into pQE30 (Qiagen) expression vector, as described elsewhere (Dong et al., 2002).

Identification of purified protein. Proteins from selected chromatographic fractions were resolved on $13 \%$ polyacrylamide/SDS gel and stained with Coomassie Brilliant Blue. Bands corresponding to the DDB activity in a Southwestern blot assay were excised from the gel and analyzed by tandem mass spectrometry (ESI-QUAD-TOF) in the Laboratory of Mass Spectrometry, Institute of Biochemistry and Biophysics (Warsaw, Poland). Resulting peptides were annotated to proteomic data bases using the Mascot software (www.matrixscience.com).

DNA binding in vitro assays. The ability of proteins to bind to damaged DNA in vitro was tested using an EMSA and a Southwestern-blot assay, as described in detail elsewhere (Pietrowska \& Widłak, 2005). Briefly, a synthetic double-stranded 36 bp-long oligonucleotide was incubated with AAAF, BPDE (Midwest Research Institute, USA) or cis-DDP (Ebewe) to generate molecular probes containing specific types of DNA damage. The treatments introduced a single lesion into $10-20 \%$ of the DNA molecules, on average (Rzeszowska-Wolny \& Widłak, 1999). Oligonucleotides were then ${ }^{32} \mathrm{P}$-end-labeled and purified 
from a $6 \%$ polyacrylamide gel. For the EMSA test radioactive oligonucleotides ( $25 \mathrm{ng}$ ) were incubated with proteins $(5 \mu \mathrm{g}$ of nuclear extracts or corresponding amounts of purified fractions) for $30 \mathrm{~min}$ at $4^{\circ} \mathrm{C}$ in buffer consisting of $20 \mathrm{mM}$ Tris $/ \mathrm{HCl}(\mathrm{pH}$ 7.6), $5 \mathrm{mM} \mathrm{MgCl}$, $0.5 \mathrm{mM}$ EDTA, $1 \mathrm{mM}$ DTT, $5 \%$ glycerol, $150 \mathrm{mM} \mathrm{NaCl}$, in the presence of competitor non-radioactive homologous oligonucleotides. Protein-oligonucleotide complexes were resolved by electrophoresis on a $6 \%$ polyacrylamide gel in $0.5 \times$ Tris/borate/EDTA buffer, and gels were dried and autoradiographed. For Southwestern-blot analysis proteins $(50 \mu \mathrm{g}$ of nuclear extracts or corresponding amounts of purified fractions) were separated on $13 \%$ polyacrylamide/SDS gels and electrotransferred onto PVDF membranes. Membrane-bound proteins were renatured and complexed with radioactive oligonucleotides (250 ng) at $25^{\circ} \mathrm{C}$ in a buffer consisting of $25 \mathrm{mM}$ Tris $/ \mathrm{HCl}$ ( $\mathrm{pH} 7.6), 100 \mathrm{mM} \mathrm{NaCl}, 1 \mathrm{mM}$ EDTA, $1 \mathrm{mM}$ DTT, $5 \mathrm{mM} \mathrm{MgCl}$ and $0.25 \%$ bovine serum albumin, in the presence of an 80-fold excess of non-radioactive non-specific competitor (E. coli DNA). After washing with the same buffer filters were autoradiographed.

\section{RESULTS AND DISCUSSION}

Nuclear extracts from rat liver cells were analysed for the presence of proteins that bind to damaged DNA using an electrophoretic mobility shift assay. The radio-labeled oligonucleotide damaged by AAAF or cis-DDP was used as a probe, and complexes with proteins were formed in the presence of excess of non-radioactive oligonucleotide, either non-damaged or damaged with AAAF or cis-DDP, as a homologous competitor. The results in Fig. 1 show that AAAF-damaged DNA is specifically bound by AAAF/DDP-DDB protein complex (lane 1), whereas cis-DDP-damaged DNA is bound by HMGB1 and AAAF/DDP-DDB protein complexes (lane 4). Accordingly, the presence of AAAF-damaged competitor DNA inhibits the probe binding by AAAF/DDP-DDB protein but not HMGB1 (lanes 3 and 6), while the presence of cisDDP-damaged competitor DNA inhibits the probe binding by both AAAF/DDP-DDB protein and HMGB1 (lanes 2 and 5) (the identity of HMGB1 in appropriate complex was confirmed in our earlier work; Łanuszewska \& Widłak, 2000).

Aiming to identify the AAAF/DDP-DDB protein we attempted to purify it to homogeneity from the chromatin fraction of rat liver cells. Proteins were fractionated by column chromatography on hydroxyapatite and CM-Sephadex, and then activity of the AAAF/DDP-DDB protein was detected in such fractions (Fig. 2). When proteins were eluted

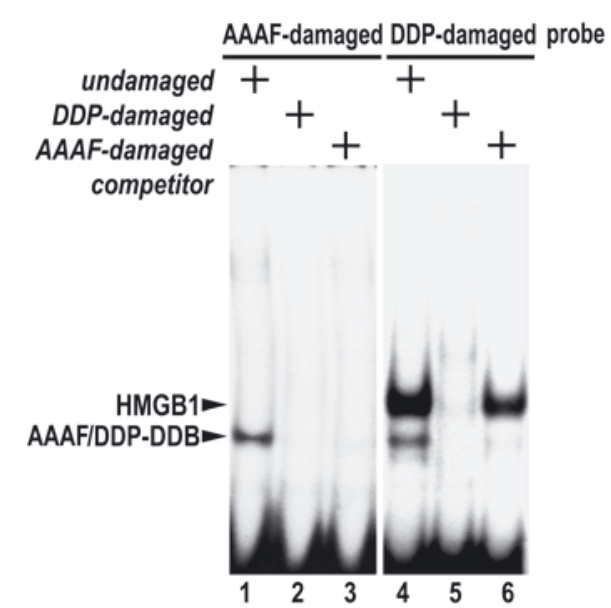

Figure 1. The major rat liver protein that recognizes DNA damaged by AAAF also binds to DNA damaged by cis-DDP and has been termed AAAF/DDP-DDB.

Complexes were formed between labeled oligonucleotide, damaged either with AAAF or cis-DDP, and nuclear extracts from rat liver cells, in the presence of a 50-fold excess of homologous non-radioactive competitor (either undamaged or damaged with AAAF or cis-DDP), and then separated by native polyacrylamide gel electrophoresis.

from hydroxyapatite-bound chromatin material by increasing $\mathrm{NaCl}$ concentrations, the AAAF/DDPDDB activity could be detected mostly in a fraction eluted with 0.4-0.45 M NaCl (lane 3). This fraction was then applied onto a CM-Sephadex column and proteins were eluted with $0.2,0.6$ and $1 \mathrm{M} \mathrm{NaCl}$. The AAAF/DDP-DDB activity was detected in the fraction eluted with $0.6 \mathrm{M} \mathrm{NaCl}$ (lane 9). This protein fraction contained two major protein species of about 25 and $18 \mathrm{kDa}$ (Fig. 2A). Southwestern-blot analysis (Fig. 2C) revealed that both proteins form in vitro complexes with an AAAF-damaged DNA probe but not with a control non-damaged probe. However, only the $25 \mathrm{kDa}$ protein form in vitro complexes with a cis-DDP-damaged probe, and we assumed that this protein band corresponds to the AAAF/DDP-DDB protein. This protein was excised from a Coomassie-stained gel and subjected to mass spectrometry analysis. The protein was identified as mitochodrial transcription factor A (TFAM, mtTFA) (identified peptides covered $39 \%$ of the mature form of rat TFAM). Similarly, the AAAF/DDP-DDB protein was purified from chromatin fraction of mouse liver cells using the same procedure and also identified as TFAM (identified peptides covered $53 \%$ of the mature form of mouse TFAM), which fully confirmed its identity. We also tried to identify the 18 $\mathrm{kDa}$ protein. However, the obtained peptides did not match any protein in proteomic data bases. In addition, the $18 \mathrm{kDa}$ protein was eluted from a gel and renatured but it did not form any complexes with either AAAF-damaged or cis-DDP-damaged DNA (not shown). 

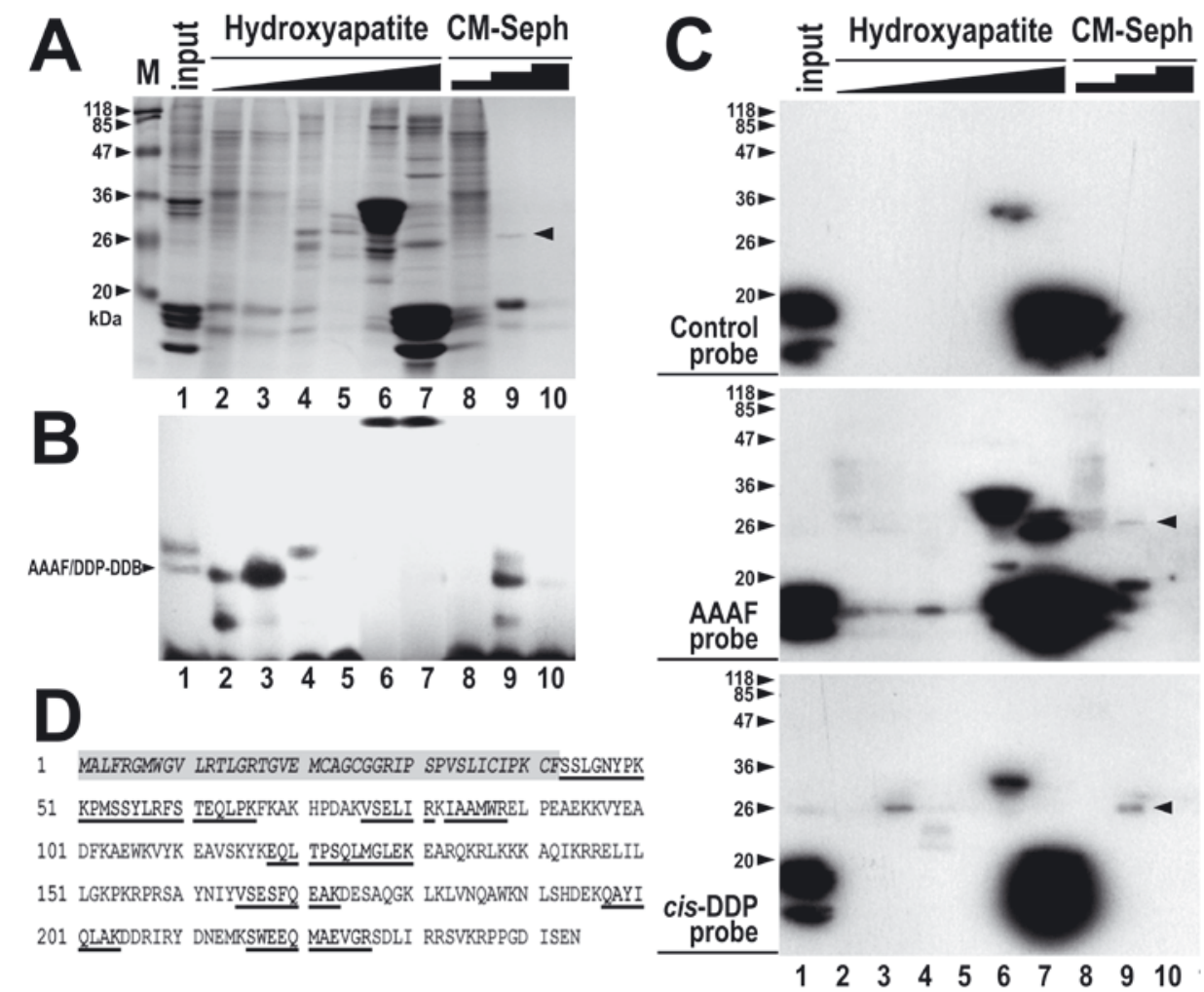

Figure 2. The AAAF/DDP-DDB protein was purified and identified as TFAM.

Panel A. SDS/PAGE analysis of chromatographically fractionated chromatin preparation from rat liver. Proteins were first eluted from a hydroxyapatite column with $0.4,0.45,0.5,0.6,0.8$ and $2 \mathrm{M} \mathrm{NaCl}$ (lanes 2-7), then the $0.45 \mathrm{M}$ fraction (lane 3) was loaded onto a CM-Sephadex column and proteins eluted with 0.2, 0.6 and $1 \mathrm{M} \mathrm{NaCl} \mathrm{(lanes} \mathrm{8-10).} \mathrm{Panel} \mathrm{B.}$ Detection of the AAAF/DDP-DDB activity in chromatographic fractions. Complexes were formed with radio-labeled cisDDP-damaged probe in the presence of genomic E. coli DNA competitor. Panel C. Southwestern-blot analysis of chromatographic fractions. A protein band corresponding to the AAAF/DDP-DDB activity is marked with an arrowhead. Panel D. The sequence of amino-acid residues of rat TFAM precursor. The mitochondrial localization region (MLR) removed from the mature protein is highlighted in gray. Peptides identified by mass spectrometry analysis of the AAAF/ DDP-DDB protein are underlined.

We have observed that complexes formed by recombinant rat TFAM with damaged DNA have the same electrophoretic mobility as complexes formed by the AAAF/DDP-DDB protein isolated from rat liver extracts (Fig. 3A). We have also aimed to study the affinity of bacterially expressed recombinant TFAM to DNA containing different types of bulky adducts. Recombinant rat TFAM was incubated with a cis-DDP-damaged radio-labelled probe in the presence of increasing amounts of different homologous competitors (Fig. 3B). The affinity of recombinant rat TFAM for AAAF-damaged or cis-DDP-damaged non-radioactive competitor was at least 33-fold greater than that for undamaged DNA (i.e. $0.06 \mu \mathrm{g}$ of AAAF-damaged oligonucleotide was as good competitor as $2 \mu \mathrm{g}$ of undamaged one). The affinity of recombinant TFAM for BPDE-damaged competitor was only 2to 4-fold higher than that for the undamaged one, which was in full agreement with previously reported characteristics of the AAAF/DDP-DDB protein (Pietrowska \& Widłak, 2005). Similarly, purified recombinant human TFAM has strong affinity

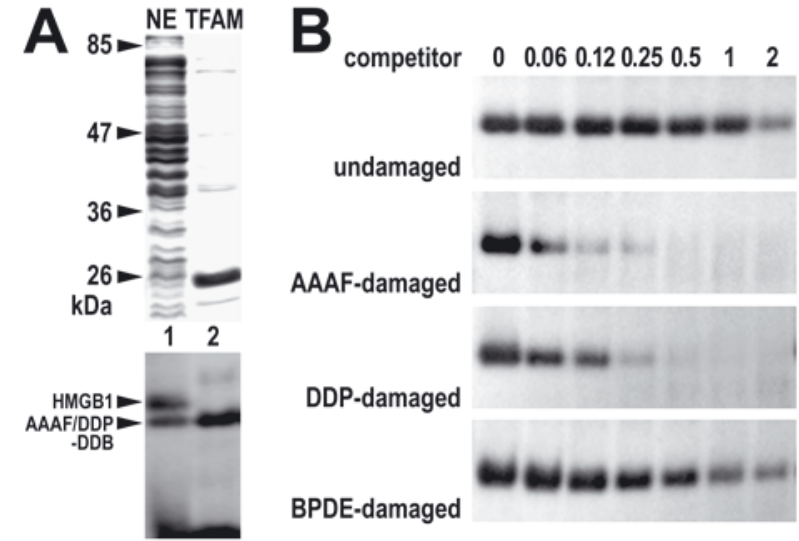

Figure 3. Bacterially expressed recombinant rat TFAM binds with high affinity to DNA damaged by AAAF.

Panel A. Nuclear extract (NE) from rat liver cells (lane 1) and recombinant rat TFAM protein (lane 2) were analyzed by SDS/PAGE (top) and EMSA with radioactive cisDDP-damaged probe (bottom). Panel B. Complexes were formed between recombinant rat TFAM and radio-labeled cis-DDP-damaged probe (25 ng), in the presence of increasing amounts of homologous non-radioactive competitor $(0.06$ to $2 \mu \mathrm{g}$, as indicated), either undamaged or damaged with AAAF, cis-DDP or BPDE. 
for AAAF-damaged or cis-DDP-damaged, but not for BPDE-damaged DNA.

Mitochondrial transcription factor A is a protein that contains two HMG-boxes, and its primary function is to regulate replication and transcription of mitochondrial DNA (Larsson et al., 1998). This protein has been previously reported to bind with enhanced affinity to DNA containing either lesions induced by cis-DDP or 8-oxo-guanine (Ohndorf et al., 1997; Yoshida et al., 2002), yet its binding to other types of DNA damage was not reported. Though mitochondria are the primary location of TFAM, this protein is detected in other cellular compartments as well. In fact, high levels of TFAM have been detected in nuclei of rat hepatoma cells (Dong et al., 2002). We have compared levels of the AAAF/DDP-DDB activity in the nuclear fraction of liver cells homogenized in the presence of Nonidet P40 and in fractions of liver cells homogenized and lysed in the absence of a detergent (this latter procedure is more likely to protect the integrity of mitochondrial membranes). In fact, we have observed that the level of the AAAF/ DDP-DDB protein was 3-4-fold lower in nuclear extracts from liver cells lysed in the absence of detergent as compared to extracts from detergent-treated cells, and that the AAAF/DDP-DDB protein could be detected in mitochondrial extracts (not shown). This indicates that during cell lysis a fraction of TFAM released from mitochondria disrupted with detergent apparently binds to chromatin and could be recovered from nuclear extracts and chromatin fractions. However, it seems possible that a fraction of TFAM that recognizes damaged DNA is also located in intact nuclei of rat hepatocytes. Although TFAM is ubiquitously expressed in rat tissues, the activity of AAAF/DDP-DDB protein was markedly lower in extracts from testes as compared to liver and other somatic tissues (Pietrowska \& Widłak, 2005). An additional nuclear isoform of TFAM (ts-HMG), produced from transcripts that have an alternative first exon, was detected in testes of mice (Larsson et al., 1996), but not testes of rats (Rantanen et al., 2001). However, the level of "primary" TFAM was markedly downregulated in both mouse and rat testes, reflecting the progress of spermatogenesis. Thus the observed reduction of the AAAF/DDP-DDB activity in testicular extracts (Pietrowska \& Widłak, 2005) apparently reflects downregulation of TFAM protein during spermatogenesis.

TFAM, like other HMG-box containing proteins, can recognize distortions in DNA structure that facilitate its physiological binding to the D-loop region in mtDNA. This feature apparently enables binding of TFAM to unwound or bent sequences at a site of DNA damage. Similar to other HMG-box proteins (e.g. HMGB1), TFAM binds with high affinity to DNA damaged by cisplatin (Ohndorf et al., 1997;
Yoshida et al., 2002). In marked contrast, however, we have shown here that TFAM binds to DNA damaged by AAAF with a much higher affinity as compared to HMGB1. The presence of Pt-DNA 1,2-intrastrand crosslinks, the major type of cisplatin-induced adducts, or dG-C8-AAF, the major type of AAAF-induced adducts, somehow differently distorts the structure of double-stranded DNA (reviewed in Łanuszewska \& Widłak, 2000). Although recognition of DNA distorted at a site of cisplatin-induced crosslinks is the general feature of the HMG-box, recognition of DNA containing other bulky damage differently changing the structure of DNA may be characteristic for a particular HMG-box protein.

The physiological consequence of TFAM binding to damaged DNA remains elusive at the moment. The protein is essential for mtDNA maintenance and possibly involved in its repair (Larsson et al., 1998). Mitochondrial DNA is highly susceptible to DNA damage, especially oxidative damage caused by byproducts of the electron transfer reactions in mitochondria. Interestingly, TFAM but not HMGB1 shows high affinity for mismatches containing 8-oxo-guanine, the major type of oxidative base modification, that suggested its involvement in recognition and repair of mtDNA damage (Yoshida et al., 2002). Homozygous disruption of the Tfam gene resulted in increased apoptosis in vivo, and most probably observed cell deaths resulted from lack of mtDNA expression and respiratory chain dysfunctions, yet more direct effects of TFAM should not be excluded (Wang et al., 2001). Interestingly, TFAM interacts directly with p53 protein, whose fraction relocalized to mitochondria at the onset of DNA damage-induced apoptosis, and the presence of p53 enhanced TFAM binding to cisplatin-damaged DNA yet inhibited its binding to 8-oxo-guanine-containing DNA (Yoshida et al., 2003). Hypothetically, TFAM that bound to damaged DNA might either facilitate or delay DNA repair (by recruiting repair complexes or shielding a lesion, respectively). Interactions with p53 in cells exposed to genotoxic agents might affect binding of TFAM to particular types of DNA damage to modulate their repair and induction of apoptosis, as proposed by Kohno and coworkers (Yoshida et al., 2003). According to our earlier data the AAAF/DDP-DDB protein, identified here as TFAM, is the major protein present in somatic tissues of rodents that recognizes and binds to DNA containing damage induced by AAAF (Pietrowska \& Widłak, 2005). The data presented here indicate the possibility that a fraction of TFAM protein that has an activity of AAAF/DDP-DDB protein, exists in nuclei of rat somatic cells. Considering the abundance of this protein and its high affinity for DNA alterations induced by AAAF, TFAM might essentially impede recognition and repair of DNA adducts induced by 
2-AF derivatives and contribute to their carcinogenic potency.

\section{Acknowledgements}

We thank Professor Samson T. Jacob for a generous gift of the rat recombinant TFAM expression vector and Professor Kimitoshi Kohno for a generous gift of the human recombinant TFAM expression vector.

This work was supported by the Ministry of Science, Grants 3P05A10524 and 2P05B12630.

\section{REFERENCES}

Dong X, Ghoshal K, Majumder S, Yadav SP, Jacob ST (2002) Mitochondrial transcription factor $\mathrm{A}$ and its downstream targets are up-regulated in a rat hepatoma. J Biol Chem 277: 43309-43318.

Heflich RH, Neft RE (1994) Genetic toxicity of 2acetylaminofluorene, 2-aminofluorene and some of their metabolites and model metabolites. Mutat Res 318: 73-114.

Kroese ED, van de Poll ML, Mulder GJ, Meerman JH (1988) The role of N-sulfation in the N-hydroxy-2acetylaminofluorene-mediated outgrowth of diethylnitrosamine-initiated hepatocytes to gamma-glutamyltranspeptidase-positive foci in male rat liver. Carcinogenesis 9: 1953-1958.

Larsson NG, Garman JD, Oldfors A, Barsh GS, Clayton DA (1996) A single mouse gene encodes the mitochondrial transcription factor A and a testis-specific nuclear HMG-box protein. Nat Gen 13: 296-302.

Larsson NG, Wang J, Wilhelmsson H, Oldfors A, Rustin P, Lewandoski M, Barsh GS, Clayton DA (1998) Mitochondrial transcription factor A is necessary for mtDNA maintenance and embryogenesis in mice. Nat Genet 18: 231-236.

Łanuszewska J, Widłak P (2000) High mobility group 1 and 2 proteins bind preferentially to DNA that contains bulky adducts induced by benzo(a)pyrene diol epoxide and $\mathrm{N}$-acetoxy-acetylaminofluorene. Cancer Lett 158: 17-25.

McGregor WG, Wei D, Chen RH, Maher VM, McCormick JJ (1997) Relationship between adduct formation, rates of excision repair and the cytotoxic and mutagenic effects of structurally-related polycyclic aromatic carcinogens. Mutat Res 376: 143-152.

Ohndorf UM, Whitehead JP, Raju NL, Lippard SJ (1997) Binding of tsHMG, a mouse testis-specific HMG-domain protein, to cisplatin-DNA adducts. Biochemistry 36: 14807-14815.

Pietrowska M, Widłak P (2005) Characterization of a novel protein that specifically binds to DNA modified by Nacetoxy-acetylaminofluorene and cis-diammine-dichloro-platinum. Acta Biochim Polon 52: 867-874.
Pietrowska M, Łanuszewska J, Walter Z, Rzeszowska-Wolny J, Widłak P (2000) Detection and characterization of rat protein recognizing DNA damaged by $\mathrm{N}$-acetoxyacetylaminofluorene. Cell Mol Biol Lett 5: 423-431.

Pil PM, Lippard SJ (1992) Specific binding of chromosomal protein HMG1 to DNA damaged by the anticancer drug cisplatin. Science 256: 234-237.

Poirier MC, Fullerton NF, Kinouchi T, Smith BA, Beland FA (1991) Comparison between DNA adduct formation and tumorigenesis in livers and bladders of mice chronically fed 2-acetylaminofluorene. Carcinogenesis 12: 895-900.

Rantanen A, Jansson M, Oldfors A, Larsson NG (2001) Downregulation of Tfam and mtDNA copy number during mammalian spermatogenesis. Mamm Genome 12: 787-792.

Reeves R, Adair JE (2005) Role of high mobility group (HMG) chromatin proteins in DNA repair. DNA Repair 4: 926-938.

Rzeszowska-Wolny J, Widlak P (1999) Damaged DNA-binding proteins: recognition of N-acetoxy-acetylaminofluorene-induced DNA adducts. Acta Biochim Polon 46: 173-180.

Sancar A, Lindsey-Boltz LA, Unsal-Kacmaz K, Linn S (2004) Molecular mechanisms of mammalian DNA repair and the DNA damage checkpoints. Annu Rev Biochem 73: 39-85.

Tang MS, Bohr VA, Zhang XS, Pierce J, Hanawalt PC (1989) Quantification of aminofluorene adduct formation and repair in defined DNA sequences in mammalian cells using the UVRABC nuclease. J Biol Chem 264: 14455-14462.

Wang J, Silva JP, Gustafsson CM, Rustin P, Larsson NG (2001) Increased in vivo apoptosis in cells lacking mitochondrial DNA gene expression. Proc Natl Acad Sci USA 98: 4038-4043.

Widlak P, Pietrowska M, Łanuszewska J (2006) The role of chromatin proteins in DNA damage recognition and repair. Histochem Cell Biol 125: 119-126.

Widłak P, Rzeszowska-Wolny J (1999) Nuclear matrix and nucleotide excision repair: damage-recognition proteins are not constitutive components of the nuclear matrix. In: Gene Therapy and Molecular Biology (Boulikas T, ed) vol 4, pp 275-284. Gene Therapy Press, Palo Alto.

Wood RD (1999) DNA damage recognition during nucleotide excision repair in mammalian cells. Biochimie $\mathbf{8 1}$ 39-44.

Yoshida $\mathrm{Y}$, Izumi H, Ise T, Uramoto $\mathrm{H}$, Torigoe $\mathrm{T}$, Ishiguchi H, Murakami T, Tanabe M, Nakayama Y, Itoh H, Kasai H, Kohno K (2002) Human mitochondrial transcription factor A binds preferentially to oxidatively damaged DNA. Biochem Biophys Res Commun 295: 945-951.

Yoshida $Y$, Izumi $\mathrm{H}$, Torigoe $\mathrm{T}$, Ishiguchi $\mathrm{H}$, Itoh $\mathrm{H}$, Kang D, Kohno K (2003) p53 physically interacts with mitochondrial transcription factor $\mathrm{A}$ and differentially regulates binding to damaged DNA. Cancer Res 63: 3729-3734. 